\title{
Parallel Performance of Mandelbrot Set Generation Using MPI On Raspberry Pi 2 Beowulf Cluster
}

\author{
Erdem Ağbahca* \\ Department of Computer Engineering, Selçuk University, Konya, Turkey
}

\begin{tabular}{|c|c|}
\hline \multicolumn{2}{|c|}{ Department of Computer Engineering, Selçuk University, Konya, Turkey } \\
\hline Article history & Performance of applications which solves problems that can be \\
\hline $\begin{array}{l}\text { Received: } \\
05.06 .2016\end{array}$ & $\begin{array}{l}\text { divided into non-sequential subtasks is greatly enhanced by running } \\
\text { every subtask on multiple cores or multiple CPUs over a cluster }\end{array}$ \\
\hline $\begin{array}{l}\text { Received in revised form: } \\
30.07 .2016\end{array}$ & $\begin{array}{l}\text { using Message-Passing Interface (MPI). These clusters can be built } \\
\text { with any type of computer from commodity computers to special }\end{array}$ \\
\hline Acc & HPC hardware, yet single board computers (SBC) like Raspberry \\
\hline 31.07 .2016 & $\begin{array}{l}\text { Pi would still be amongst the lowest power consuming and the } \\
\text { most mobile considering Pi's small volume and passive cooling. }\end{array}$ \\
\hline Key words: & Mostly valued by hobbvists, Raspberry $\mathrm{Pi}$ is a popular SBC used in \\
\hline $\begin{array}{l}\text { Message Passing Interface, } \\
\text { Raspberry Pi, Parallel } \\
\text { Programming, Cluster }\end{array}$ & $\begin{array}{l}\text { different types of projects from home automation to wearable } \\
\text { gadgets and small servers to auto-navigating robots. At the same }\end{array}$ \\
\hline Computing & $\begin{array}{l}\text { time, Raspberry Pi's can still be a part of computer cluster since it } \\
\text { runs many operating systems available on the market, furthermore } \\
\text { the most supported one is Raspbian which is a Debian derivative. } \\
\text { In this study, we presented timing records of test results for } \\
\text { calculating Mandelbrot set for visual depiction over various process } \\
\text { counts on a nine node Raspberry Pi } 2 \text { Beowulf cluster. }\end{array}$ \\
\hline
\end{tabular}

\footnotetext{
* Correspondence: agbahcaerdem@gmail.com
} 


\section{Introduction}

MPI is a de-facto standard portable message-passing system, addressing parallel programming model with its dynamic process spawning, data movement between processes, parallel $\mathrm{I} / \mathrm{O}$, collective operations and remote-memory access operations [1]. MPI is used to distribute subtasks among a computer network and by doing this, it reduces the time to complete the whole task.

Raspberry $\mathrm{Pi}$ is a well-known $\mathrm{SBC}$ which is used in different types of mostly non-CPU intensive projects like; home automation, arcade boxes, TV and jukeboxes, autonomous robots, home-type servers. There are several studies conducted at Texas A\&M University [2], Southampton University [3] and Boise State University [4] to prove Raspberry Pi's usefulness for cluster computing on non-CPU intensive fields and educational purposes as a low power and low budget alternative to current clusters. Considering Raspberry $\mathrm{Pi} 2$ Model $\mathrm{B}$ is equipped with stock overclocked $900 \mathrm{MHz}$ quad-core ARM Cortex-A7 CPU and 1 GB of ram, these SBCs can provide enough computational power for many problems when clustered together. In this study, 9 Raspberry Pi $2 \mathrm{~s}$ are overclocked to 1000 $\mathrm{MHz}$ and $16 \mathrm{~GB}$ Class $10 \mathrm{SD}$ cards are used to provide local storage.

Computing performance is measured in floating-point operations per second (flops) and there are several tools to benchmark a system's performance. For example,
LINPACK [5] and High-Performance LINPACK are amongst the most popular and this can be seen in Iridis-Pi [3] study. However, when it comes to real life implementations, performance of a system heavily depends on algorithm of its programming which loses many flops along their design. We propose our test results in wall clock time (seconds) to provide an actual depiction for comparison of how long a problem takes to calculate along with its pseudo-code. In addition, to show that the results scale with process/node count, a problem must be both embarrassingly parallel and it should also require somewhat inter-node communication. So that, a Mandelbrot set for visualization calculation is picked. For a brief explanation, a Mandelbrot set [6] is a set of complex numbers which can be generated from the formula $z_{(n+1)}=z_{n}^{2}+c$ and when $z_{0}=0$ is used as starter, it is bounded [7].

\section{Experimental}

Since Raspberry Pi SBCs are small in volume and not demanding in perspective of power, creating a Beowulf cluster from them is fairly easy. These SBCs can gain portability when stacked like a rack as shown in Fig. 1. Powered from 5V micro USB port, these SBCs can drain up to $2 \mathrm{~A}$ per card when on full load and USB devices connected. In this study, a $5 \mathrm{~V} 40 \mathrm{~A}$ dc power supply is used to sustain further expansion of cluster size for oncoming studies.

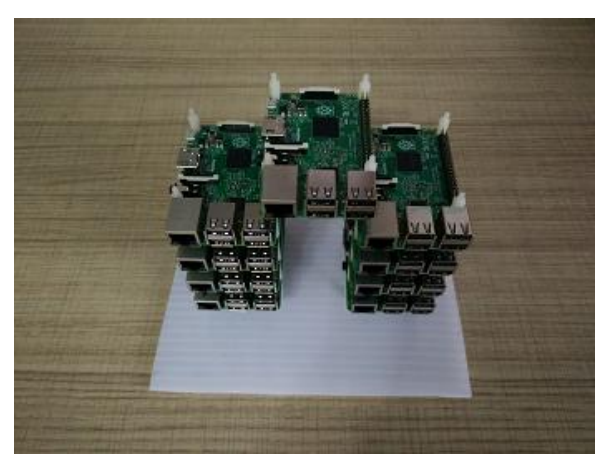

Figure 1. Raspberry Pi Stack 
Raspberry Pi's can run several different operating systems but Raspbian [8] a Debian Linux derivative is the commonly used one. These operating systems are mostly distributed as image files and that needs to be written on SD card and in this study Raspbian (Jessie) is used. After the first boot, several prerequisites are done for example, expanding the file system to SD card and overclocking the system to $1000 \mathrm{MHz}$ as depicted above. There are several popular MPI implementation libraries exists, MPICH
(Ver. 3.2) [9] is used in this study. After preparing and changing names (hostname) of each node, these SBCs should be connected over a network to form a Beowulf cluster. A 100 Mbit 3Com Switch (4400) is used to connect the nodes to university network. One of the Pi's which is the master node, connected to a monitor, keyboard and mouse and it also contains a file with every node's IP address. Architecture and the final build of the said system can be seen in Fig. 2 and Fig. 3 . respectively.

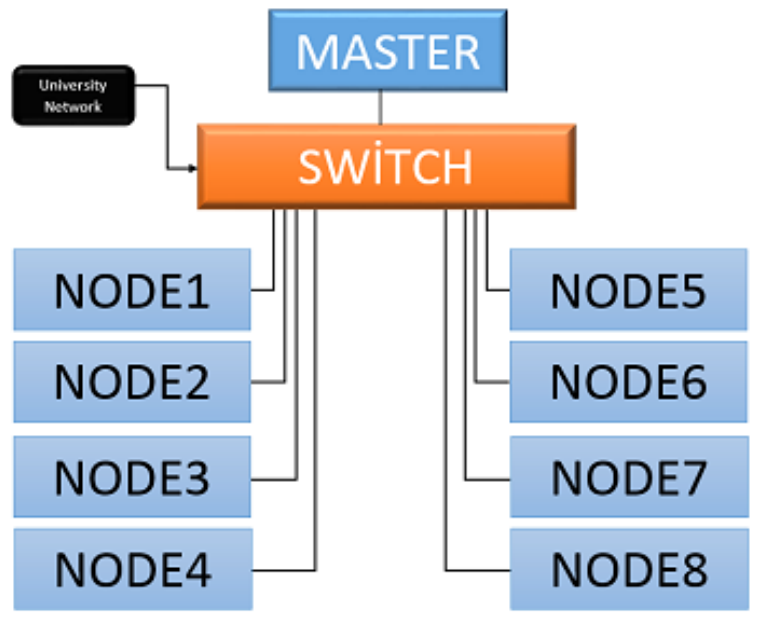

Figure 2. System Architectural Diagram

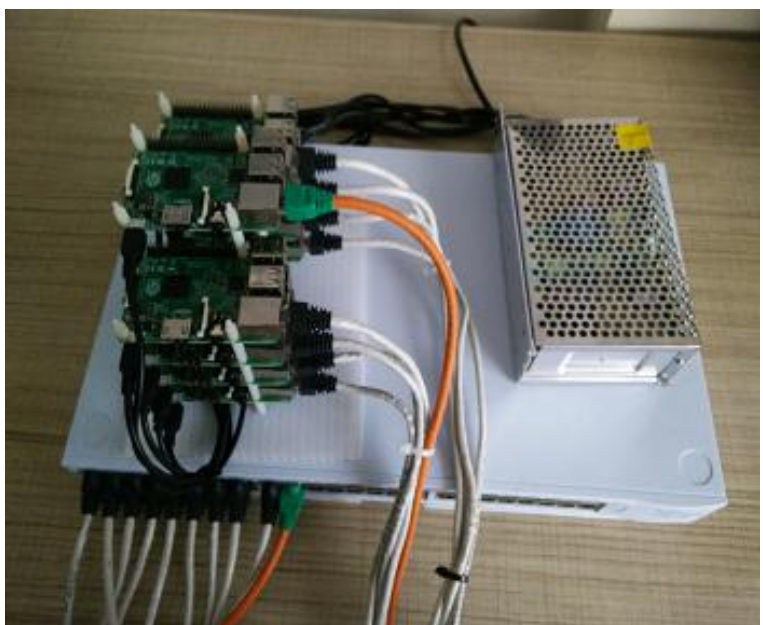

Figure 3. Actual System Image and $\mathrm{Y}$ boundaries. A program is coded in $\mathrm{C}$

Considering each Pi CPU has 4 threads, they can run 4 processes simultaneously and that leads to 36 processes in total for the 9 node cluster. A program which calculates the Mandelbrot visualization for 10080x 10080 pixel size in between $(-2,2)$ fields for both $\mathrm{X}$ using MPICH libraries then copied to every node using scp command. Task is divided depending on the $\mathrm{X}$ coordinate of the Mandelbrot visualization. So, every process iterates for all $\mathrm{Y}$ coordinates, but only 
iterates in their individual $X$ limits. Measurement of the wall clock time starts after MPI initialization and ends when all of the pixels are calculated, just before they are written to a file. Because of SD card speed concerns, this measurement does not include writing pixels to a ppm file. Pseudo-core for the algorithm can be seen in Fig. 4.

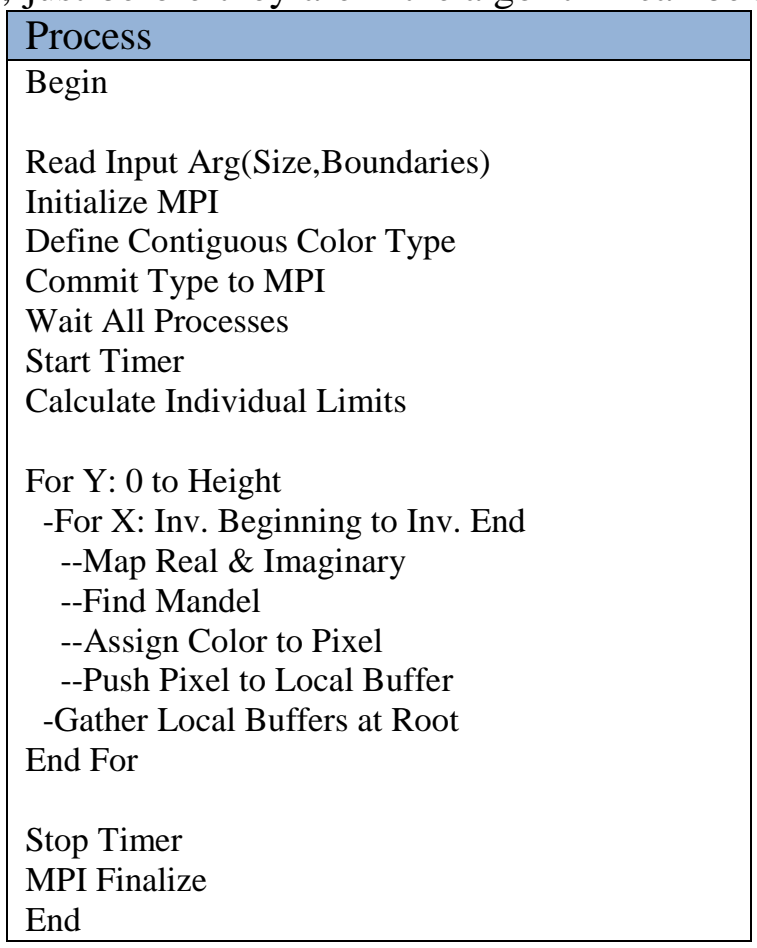

\section{Results and Discussion}

Time results are gathered for $1,4,8$, $12, \ldots, 32,36$ processes. 1 process means totally serial and every 4 process means computation includes 1 node. For example, when run as 12 processes, 4 processes running on each master, node1 and node2 nodes. For each process count, tests are repeated 10 times (D1, ..., D10) with a waiting period of 5 minutes in between every test to minimize ambient effects like temperature on cluster. Results are rounded to nearest integer and averages of these 10 results are used as final result. Results can be seen at Table 1.

Table 1. Time Results of Tests (Seconds)

\begin{tabular}{|c|c|c|c|c|c|c|c|c|c|c|}
\hline $\begin{array}{c}\text { Process } \\
\text { Count }\end{array}$ & $\mathbf{1}$ & $\mathbf{4}$ & $\mathbf{8}$ & $\mathbf{1 2}$ & $\mathbf{1 6}$ & $\mathbf{2 0}$ & $\mathbf{2 4}$ & $\mathbf{2 8}$ & $\mathbf{3 2}$ & $\mathbf{3 6}$ \\
\hline D 1 & 279 & 208 & 114 & 117 & 110 & 113 & 89 & 95 & 98 & 125 \\
\hline D 2 & 279 & 215 & 110 & 121 & 101 & 103 & 100 & 85 & 100 & 121 \\
\hline D 3 & 279 & 211 & 142 & 117 & 97 & 122 & 85 & 85 & 97 & 110 \\
\hline D 4 & 282 & 228 & 111 & 117 & 109 & 125 & 102 & 80 & 101 & 112 \\
\hline D 5 & 279 & 224 & 112 & 119 & 108 & 99 & 82 & 82 & 94 & 109 \\
\hline D 6 & 277 & 222 & 113 & 121 & 94 & 119 & 88 & 85 & 109 & 112 \\
\hline D 7 & 283 & 224 & 116 & 121 & 92 & 97 & 101 & 80 & 92 & 112 \\
\hline D 8 & 279 & 216 & 111 & 121 & 96 & 117 & 88 & 82 & 91 & 110 \\
\hline D 9 & 278 & 169 & 155 & 114 & 100 & 100 & 89 & 77 & 96 & 110 \\
\hline D 10 & 286 & 153 & 153 & 116 & 95 & 111 & 105 & 79 & 91 & 124 \\
\hline $\begin{array}{c}\text { AVG } \\
\text { (s) }\end{array}$ & 280 & 207 & 124 & 118 & 100 & 111 & 93 & 83 & 97 & 115 \\
\hline
\end{tabular}


When serially executed (as 1 process) in average it takes 280 seconds to calculate whole set. Raising process count to 4 reduces this time by 73 seconds and when process count is doubled to 8 , time results drops to 124 seconds, to a level nearly half of the previous. This performance boost continues until process count is risen to 20 processes. That additional node causes more communication overhead compared to its computation contribution, so that it affects performance negatively and increases the total computational time. When run as 24 and 28 processes, it both reduces the time result below all of the results that came before thus arising a positive performance boost again. Then, when run as 32 processes the time result is sat in between 20-24 process results causing a performance loss.
Even though more processing power is included, when the cluster is fully utilized it takes 114 seconds to complete the calculation, which is worse than running this problem as 16 processes, resulting a huge drawback in performance due to communication overhead. Best result is reached by running 28 processes over 7 nodes as 83 seconds. Interpretation of these results is, when the barrier of needed computational power is reached, cluster performance loses its advantage because of communication overheads. That can both be overcome by increasing the problem size or reducing the communication need. Graph of average time results of the tests can be seen in Fig. 5. and the resulting Mandelbrot image can be seen in Fig. 6.

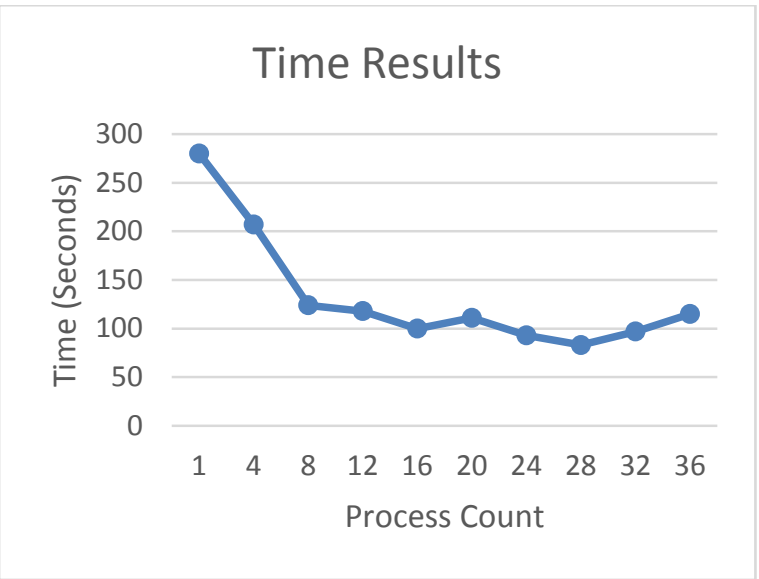

Fig 5. Average Time Results Graph

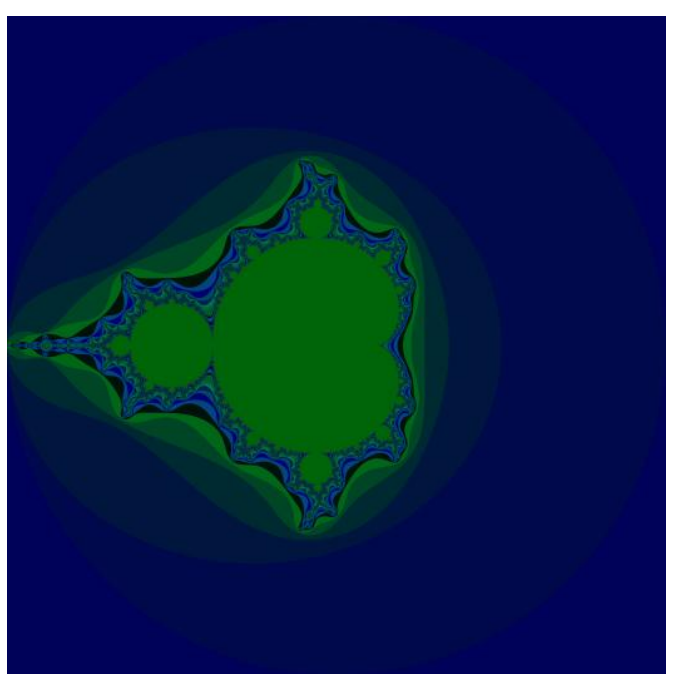

Fig 6. Resulting Mandelbrot Image 


\section{Conclusion}

Raspberry Pi's proved themselves to be useful for complex computation by clustering them together. Their small weights and small volumes provide better mobility over conventional clusters and considering their low cost and low power consumption they can be used in specific areas benefiting these properties.

Since these SBC's are not powerful in CPU perspective, when more resources thrown for computation, it doesn't compensate the communication loss of time due to communication needs of each additional process. So that, the communication overhead wildly affects the end-result. To tackle this, algorithm can be change to send the calculated results after every process is done calculating all of their local pixels instead of sending them after each $Y$ coordinate. Yet, an even better improvement can be achieved by using OpenMP to eliminate communication inside of the same node. Gather calls to respond can be reduced to 1 instead of 4 by running only 1 MPI process that talks inter-node for each node by spawning 4 threads instead of 4 MPI processes. Thus loss of time due to communication overhead can be decreased. This will be studied for future work.

\section{References}

[1] University of Tennessee, "MPI: A Message-Passing Interface Standard Version 3.1," 2015.

[2] E. Wilcox, P. Jhunjhunwala, K. Gopavaram, and J. Herrera, "Pi-
Crust: A Raspberry Pi Cluster

Implementation."

[3] S. J. Cox, J. T. Cox, R. P. Boardman, S. J. Johnston, M. Scott, and N. S. O'Brien, "Iridis-pi: a low-cost, compact demonstration cluster," Cluster Computing-the Journal of Networks Software Tools and Applications, vol. 17, no. 2, pp. 349358, Jun, 2014.

[4] J. Kiepert. "Creating a Raspberry PiBased Beowulf Cluster,"

http://coen.boisestate.edu/ece/files/20 13/05/Creating.a.Raspberry.PiBased.Beowulf.Cluster_v2.pdf.

[5] J. J. Dongarra, P. Luszczek, and A. Petitet, "The LINPACK benchmark: past, present and future," Concurrency and Computation-

Practice \& Experience, vol. 15, no. 9, pp. 803-820, Aug, 2003.

[6] X. Y. Wang, and Q. J. Shi, "The generalized Mandelbort-Julia sets from a class of complex exponential map," Applied Mathematics and Computation, vol. 181, no. 2, pp. 816-825, Oct 15, 2006.

[7] J. R. Vaughan, and G. R. Brookes, "The Mandelbrot Set as a Parallel Processing Benchmark," University Computing, vol. 11, no. 4, pp. 193197, Dec, 1989.

[8] Anonymus. "Raspberry Downloads," 21/03/2016;

https://www.raspberrypi.org/downloa ds/.

[9] Anonymus. "MPICH | HighPerformance portable MPI," 21/03/2016; https://www.mpich.org/. 